\title{
Corticosteroids versus vestibular rehabilitation in long-term outcomes in vestibular neuritis
}

\author{
Elshahat Ibrahem Ismail*, Ashraf Elsayed Morgan and Akram Mohamed Abdel Rahman \\ Department of ENT, Audiology Unit, Faculty of Medicine, Mansoura University, Egypt
}

Received 9 September 2017

Accepted 13 November 2018

\begin{abstract}
.
BACKGROUND: The management strategy for functional recovery after vestibular neuritis (VN) has not yet been established. Therapeutic choices involve corticosteroids, vestibular rehabilitation therapy (VRT) and the combination of corticosteroids with VRT.

OBJECTIVE: The present study aimed to compare the efficacy of corticosteroids, vestibular rehabilitation, and combination of them in terms of subjective and objective improvement in patients with VN.

METHODS: A prospective randomized study was conducted on 60 patients with acute vestibular neuritis within 3 days after symptom onset. The patients were divided into three groups; steroid group treated with corticosteroids $(n=20)$, VRT group $(n=20)$ managed with vestibular rehabilitation exercises and combination group $(n=20)$ received combined (corticosteroids and vestibular exercises). Groups were compared by caloric lateralization, vestibular myogenic potential amplitude asymmetry and Dizziness Handicap Inventory scores, both at presentation and up to 12 months.

RESULTS: The study found no statistically significant difference between the three groups of the study at the end of the follow up period.

CONCLUSION: Corticosteroids and VRT seem to be equivalently effective in patients with VN. The study proposes that corticosteroids may accelerate the recovery of $\mathrm{VN}$, with no more beneficial role in the long-term prognosis of the disease.
\end{abstract}

Keywords: Corticosteroids, vestibular rehabilitation therapy, vestibular neuritis

\section{Introduction}

Vestibular neuritis $(\mathrm{VN})$ is the third most common cause of peripheral vestibular vertigo. It has an annual incidence of 3.5 per 100,000 population and accounts for $7 \%$ of the patients at outpatient clinics specialized for in the treatment of vertigo [21].

The principal symptoms of $\mathrm{VN}$ are severe rotatory vertigo with apparent movement of objects in the visual field (oscillopsia), nausea, horizontally

\footnotetext{
*Corresponding author: Elshahat Ibrahem Ismail, MD, Department of ENT, Audiology Unit, Faculty of medicine, Mansoura Egypt, El-Gomhoria St., Mansoura, Postal Code 35516, Egypt. Tel.: +20 01117803079; E-mail: drshahat@yahoo.com.
}

rotating spontaneous nystagmus to the non-affected side and a tendency to fall to the affected side; these symptoms are acute in onset and persist for many days. The head impulse test shows impaired function of the vestibulo-ocular reflex when the patient turns in the direction of the affected ear. The caloric test confirms reduction or absence of excitability of the horizontal semicircular canal. Acute audiological and other neurological symptoms, especially central oculomotor signs, are absent. This is important in differentiating vestibular neuritis from central "pseudo-vestibular neuritis" [22].

Clinical recovery is achieved via the restoration of peripheral labyrinthine function (which is usually 
incomplete in the case of vestibular neuritis). It is also achieved through the proprioceptive and visual substitution for the unilateral vestibular deficit combined with the gradual adaptation of the brain to the imbalance in the vestibular tone (central compensation) [24].

Several studies have reported continuous or episodic vertigo or unsteadiness in 43 to $53 \%$ of patients [1, 12]. In 1993, Okinaka et al. [16] stated that horizontal semicircular canal paresis occurred in $90 \%$ of patients approximately 1 month after the onset of symptoms and in $80 \%$ of patients approximately 6 months after the onset of symptoms, whereas the caloric responses were normalized in only $42 \%$ of them. However, it should be noted that the vestibular impairment, deriving from the results of bed-side and laboratory tests, does not reflect the subjective clinical complaints and the level of incapacity. Furthermore, up to $20 \%$ of patients with vestibular neuritis experience continued symptoms such as impaired vision and postural imbalance during walking and especially during head movements, despite the normalization of the caloric testing [20].

It should be noted that the management strategy for peripheral recovery of function after $\mathrm{VN}$ has not yet been established. The therapeutic choices involved: (1) corticosteroids, (2) antiviral therapy (acyclovir), (3) a combination of corticosteroids and an antiviral agent and (4) vestibular rehabilitation therapy (VRT).

The rationale for treating vestibular neuritis with corticosteroids is based on the previous studies which documented positive effects among patients with acute peripheral neuritis such as optic neuritis [2], idiopathic facial nerve paresis [6] and idiopathic hearing loss [18]. A study reported that treatment with valacyclovir, an antiviral agent, did not improve the outcomes and the combination of these drugs was not more effective than methylprednisolone alone [9]. Goudakos et al. [7], based on systematic review and meta-analysis, suggest that corticosteroids improve only the caloric extent and recovery of canal paresis of patients with vestibular neuritis while clinical recovery does not seem to be better in patients receiving corticosteroids.

There is a moderate to strong evidence that vestibular rehabilitation is a safe and effective management for unilateral peripheral vestibular dysfunction, based on a number of high-quality randomized controlled trials. However, there is moderate evidence that vestibular rehabilitation resolves symptoms and improves functioning in the medium term [15].
Accordingly, this prospective randomized trial was conducted in the present research to evaluate the short and long term improvement of clinical symptoms and the extent of caloric paresis among VN patients receiving corticosteroids, VRT or the combination of both with inclusion of a symptom-based outcome measure, in addition to an objective measure of vestibular improvement (caloric test).

\section{Methods}

The sample of the study is composed of patients aged 20 to 50 years old with typical picture VN. These patients were examined over a period of time of two years starting from April 2015 to April 2017 at the Audiology unit of the Faculty of Medicine in Mansoura University.

The diagnosis of vestibular neuritis was based on the following criteria. There was a history of the acute or sub acute (i.e., within minutes to hours) onset of severe, prolonged rotatory vertigo, nausea, and postural imbalance. On clinical examination, there was a normal audiogram $(250-8000 \mathrm{~Hz})$ and a normal middle ear function. Also, there was a horizontal spontaneous nystagmus with a rotational component toward the unaffected ear (fast phase) without evidence of a central vestibular lesion, and the head-thrust test (performed by turning the head of the patient rapidly to the right and left to provoke compensatory eye movements) showed an ipsilateral deficit of the horizontal semicircular canal [10]. Caloric irrigation showed hyporesponsiveness or lack of responsiveness of the horizontal canal of the affected ear. The asymmetry between the two sides should be more than 20 percent as measured with the use of Jongkees's formula for vestibular paresis [14].

Patients were excluded if they had a history of vestibular dysfunction before the acute onset of symptoms or had symptoms that began more than three days before recruitment; if they had additional cochlear symptoms, such as tinnitus or acute hearing loss before, during, or after the onset of vertigo; if they had inferior vestibular neuritis; if they had central ocular motor dysfunction or central vestibular dysfunction; if they had other signs or symptoms of brain-stem or cerebellar disorders, abnormal findings on magnetic resonance imaging of the brain stem or cerebellum in diffusion-weighted images or of hyperintense lesions in T2-weighted images in combination with contrast enhancement in T1-weighted 
images, a history of psychiatric disorders, glaucoma, ongoing infection, severe diabetes mellitus (a fasting blood glucose level $>180 \mathrm{mg}$ per deciliter), or severe hypertension (blood pressure $>180 \mathrm{~mm} \mathrm{Hg}$ systolic or $>110 \mathrm{~mm} \mathrm{Hg}$ diastolic); or if there were contraindications to the use of corticosteroids, such as peptic ulcer disease or known osteoporosis (on the basis of bone-density testing or a history of fracture).

\subsection{Equipment}

1. Two channel audiometer, Interacoustic AC40 diagnostic audiometer, version 1.48 (Denmark);

2. Locally made sound-treated room;

3. Immittancemetry, GSI, tympstar, middle ear analyzer version 2 with $226 \mathrm{~Hz}$ probe tone frequency (USA);

4. Videonystagmography, Micromedical, Spectrum, Visual eye, version 6.1. (USA) and

5. Biologic Auditory Evoked Potential, Navigator Pro, version 7.2.1 ((Natus Medical, Inc., San Carlos, CA, USA).

\subsection{Procedures}

During the acute stage of disease, all sample of the study received antiemetic agent (50 to $150 \mathrm{mg}$ of dimenhydrinate a day) for a maximum of three days. Within three days after the onset of symptoms, patients were randomly assigned into one of three treatment groups.

Patients in the vestibular exercises group (VRT group) were instructed to perform a vestibular rehabilitation program for 6 weeks. This program consisted of gaze stabilizing exercises VOR x $1 \&$ x 2 (in VOR x1, the patient fixes eye on a stationary object while moving the head. While in VOR $\mathrm{x} 2$, the target moves in the opposite direction to the head while the eye fixed on target); patients were also advised to perform exercises for balance and gait enhancement. Patients received written instructions and drawings describing the home exercises.

In the second group (steroid group), patients were given steroid (methylprednisolone $20 \mathrm{mg}$ tablets three times daily for one week tapered gradually over another week with $\mathrm{H} 2$ blocker once a day).

The third group (combination group) patients were instructed to perform VRT and were given steroid in a protocol similar to the previous two groups.

Patients were informed of the potential adverse effects of the medications and were asked to report them as soon as possible. At the first month of the follow-up evaluation, patients were asked about compliance to the treatment plan, and whether adverse effects had developed during the treatment or if any vertigo episodes had occurred; patients were also asked about any new symptoms that had developed during the course of the disease such as tinnitus, hearing loss, or aural fullness.

The clinical status and the improvement of our patients were assessed after an otoneurologic examination and laboratory tests at specific time points were also carried out. The investigator who performed the follow-up evaluations of outcomes was masked to the patients' allocation to the treatment groups. The baseline assessment was on the fourth day after onset, and follow-up appointments occurred at 1,3,6, and 12 months after the disease onset. The otoneurologic examination consisted of a general head and neck examination, as well as otoscopy, basic audiological evaluation, head-shaking test and head-impulse test, and tests for pathologic nystagmus (spontaneous, gaze-evoked, positional, and Dix-Hallpike maneuver). For the evaluation of postural balance and gait, the Romberg test and the Fukuda stepping test were implemented. Patients' functional, emotional, and physical handicaps were measured with the Dizziness Handicap Inventory (DHI) [13] using the Arabicversion [4].

Caloric irrigation and cervical vestibular evoked myogenic potentials (cVEMPs) were used as measures of unilateral vestibular loss. The extent of canal paresis was measured using caloric irrigation with water at $30^{\circ} \mathrm{C}$ and $44^{\circ} \mathrm{C}$. The vestibular paresis formula by Jongkees was used to calculate caloric lateralization as follows: $\left\{\left[\left(\mathrm{R} 30^{\circ}+\mathrm{R} 44^{\circ}\right)-\left(\mathrm{L} 30^{\circ}\right.\right.\right.$ $\left.\left.\left.+\mathrm{L} 44^{\circ}\right)\right] \div\left(\mathrm{R} 30^{\circ}+\mathrm{R} 44^{\circ}+\mathrm{L} 30^{\circ}+\mathrm{L} 44^{\circ}\right)\right\} \times 100$, where $\mathrm{R}$ indicates right and $\mathrm{L}$ left, and ${ }^{\circ}$ is ${ }^{\circ} \mathrm{C}$. Based on our vestibular laboratory norms, abnormal caloric findings were recorded if caloric lateralisation was $20 \%$ or higher.

The cVEMPs were recorded in response to tone burst stimuli delivered ipsilaterally at $95 \mathrm{~dB}$ with the patient in the sitting position and head turn to the opposite side. Surface electrodes were applied over the unilateral sternocleidomastoid muscle, with a reference electrode on the sternoclavicular joint and a ground electrode on the forehead. The cVEMPs were considered abnormal if they were absent or if the amplitude asymmetry ratio of the affected side to the unaffected side was $25 \%$ or higher.

Complete disease resolution was considered if the DHI score was less than 6, caloric lateralisation was less than $20 \%$, and the cVEMPs were normal. 


\subsection{Statistical analysis}

Data was analyzed using Statistical Package for IBM-SPSS computer program version 22. Quantitative parametric data were presented in mean and standard deviation, while qualitative data were presented as frequency. One way Analysis of Variance (ANOVA) was used for comparing quantitative parametric data while chi-square " $\chi 2$ " test was used to compare the qualitative data. Pearson's correlation coefficient test was used to correlate different variables. $P$ value less than 0.05 was considered statistically significant.

\section{Results}

During the two years of the study protocol, 95 patients with symptoms of acute VN were seen in the Audiology unit at Mansoura University Hospital. 84 patients met the inclusion criteria and consented to participate in the study. Only 20 patients in each of the three study groups completed full follow up (24 patients dropped out at 6 and 12 months of follow up. Besides, some of the patients refused follow up and other informed us by phone that they became well and did not need further evaluation). Table (1) summarizes the demographic characteristics of the included patients. No statistically significant differences among the groups were found in the mean age, the sex ratio or the lesion side.

As shown in Table (2), the extent of vestibular paresis, as calculated with the formula by Jongkees, did not differ significantly among the study groups at the baseline evaluation. At the first month followup visit, the three study groups showed significant canal improvement without statistically significant

Table 1

Comparison among the study groups in terms of age, sex, and side affected

\begin{tabular}{llcccc}
\hline Items & $\begin{array}{c}\text { VRT group } \\
(n=20)\end{array}$ & $\begin{array}{c}\text { Steroid group } \\
(n=20)\end{array}$ & $\begin{array}{c}\text { Combination group } \\
(n=20)\end{array}$ & $P$ \\
\hline Age & Mean & 49.3 & 47.9 & 49.1 & 0.9 \\
& ASD & 11.6 & 13.7 & 12.8 & \\
\multirow{4}{*}{ Sex } & Range & $20-65$ & $23-59$ & $20-61$ & 0.6 \\
& Male & $9(45 \%)$ & $12(60 \%)$ & $10(50 \%)$ & 0.9 \\
& Female & $11(55 \%)$ & $8(40 \%)$ & $10(50 \%)$ & \\
& Right & $12(60 \%)$ & $11(55 \%)$ & $11(55 \%)$ & 0.9 \\
& Left & $8(40 \%)$ & $9(45 \%)$ & $9(45 \%)$ & \\
\hline
\end{tabular}

Data expressed as mean \pm SD or as frequency (number-percent). SD: standard deviation; $P$ : Probability *: significance $<0.05$. Test used: one way ANOVA -test for data expressed as mean \pm SD and Pearson's chi-square for data expressed as frequency.

Table 2

Comparison of unilateral caloric weakness (UCW) among the study groups at different periods of time starting from the study till 12 months follow up

\begin{tabular}{|c|c|c|c|c|c|}
\hline \multicolumn{2}{|c|}{$\begin{array}{l}\text { Duration of } \\
\text { evaluation }\end{array}$} & \multirow{2}{*}{$\begin{array}{c}\begin{array}{c}\text { VRT group } \\
(n=20)\end{array} \\
69.20\end{array}$} & \multirow{2}{*}{$\begin{array}{c}\begin{array}{c}\text { Steroid group } \\
(n=20)\end{array} \\
70.90\end{array}$} & \multirow{2}{*}{$\begin{array}{c}\begin{array}{c}\text { Combination group } \\
(n=20)\end{array} \\
69.50\end{array}$} & \multirow{2}{*}{$\begin{array}{c}\mathrm{P} 1 \\
0.96\end{array}$} \\
\hline Baseline & Mean & & & & \\
\hline & $\pm \mathrm{SD}$ & 20.70 & 19.10 & 21.60 & \\
\hline \multirow[t]{2}{*}{1 month } & Mean & $52.10^{\mathrm{a}}$ & $43.90^{\mathrm{a}}$ & $40.80^{\mathrm{a}}$ & 0.26 \\
\hline & $\pm \mathrm{SD}$ & 17.30 & 24.40 & 24.60 & \\
\hline \multirow[t]{2}{*}{3 months } & Mean & $30.20^{\mathrm{ab}}$ & $29.10^{\mathrm{ab}}$ & $28.80^{\mathrm{ab}}$ & 0.9 \\
\hline & $\pm \mathrm{SD}$ & 11.10 & 10.90 & 12.30 & \\
\hline \multirow[t]{2}{*}{6 months } & Mean & $26.30^{\mathrm{ab}}$ & $24.50^{\mathrm{ab}}$ & $24.20^{\mathrm{ab}}$ & 0.8 \\
\hline & $\pm \mathrm{SD}$ & 11.90 & 12.70 & 10.30 & \\
\hline \multirow[t]{3}{*}{12 months } & Mean & $15.80^{\mathrm{abc}}$ & $19.10^{\mathrm{abc}}$ & $14.20^{\mathrm{abc}}$ & 0.14 \\
\hline & $\pm \mathrm{SD}$ & 7.700 & 8.900 & 6.900 & \\
\hline & $\mathrm{P} 2$ & $<0.001 *$ & $<0.001 *$ & $<0.001 *$ & \\
\hline
\end{tabular}

SD: standard deviation P: Probability *: significance $<0.05$. Test used: for comparison between groups one way ANOVA -test (P1) \& for comparison between time intervals repeated measures ANOVA followed by post-hoc Bonferroni (P2). ${ }^{a}$ : significance relative to baseline either in VRT group, steroid group or combination group. ${ }^{\mathrm{b}}$ : significance relative to 1 month either in VRT group, steroid group or combination group. ${ }^{c}$ : significance relative to 3 months either in VRT group, steroid group or combination group. ${ }^{\mathrm{d}}$ : significance relative to 6 months either in VRT group, steroid group or combination group. 
Table 3

Number of patients with abnormal VEMP Response in the study groups at different duration from the start of the study till 12 months follow up

\begin{tabular}{lccc}
\hline $\begin{array}{l}\text { Duration of } \\
\text { evaluation }\end{array}$ & $\begin{array}{c}\text { VRT group } \\
(n=20)\end{array}$ & $\begin{array}{c}\text { Steroid group } \\
(n=20)\end{array}$ & $\begin{array}{c}\text { Combination } \\
\text { group } \\
(n=20)\end{array}$ \\
\hline Baseline & 6 & 8 & 8 \\
1 month & 6 & 6 & 6 \\
3 months & 4 & 3 & 2 \\
6 months & 1 & 0 & 0 \\
12 months & 0 & 0 & 0 \\
\hline
\end{tabular}

differences among the study groups. During the repeated follow up visits, the study groups kept the improvement pattern. However, no significant difference was observed among the study groups.

At the baseline evaluation, otolith dysfunction (as measured by the cVEMPs) was observed in 6 patients of the VRT group and 8 patients in both the steroid and combination groups (Table 3). At the first month follow-up visit, 2 patients in each steroid and combination group had recovered from otolith dysfunction, while patients in the VRT group maintained abnormal VEMPs. Almost complete otolith function could be regained, at 6 months interval, from the disease onset regardless of the method of treatment.

Patient handicaps, as measured with the DHI, showed no statistically significant differences among the three study groups during baseline evaluation,

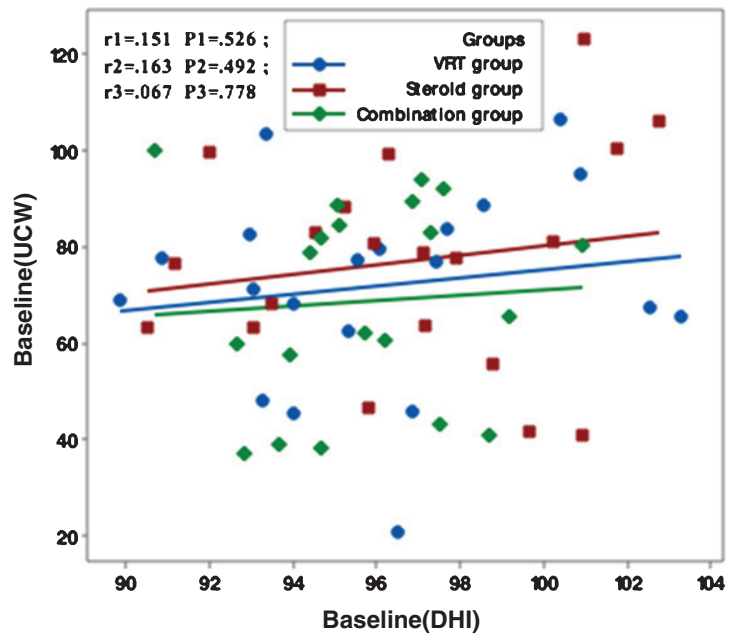

Fig. 1. Correlation between UCW and DHI in the study groups at the start of the study. $r$ : Pearson's correlation coefficient; P: Probability; *: significance $<0.05$.

after one month of treatment and during 3, 6 and 12 months follow-up (Table 4). On the other hand, there was highly statistically significance in DHI scores at one month and during the follow up periods in each of the study group separately.

Figures 1-5 showed no significant correlation between unilateral caloric weakness (UCW) and DHI in any of studied groups from start of evaluation till the end of the study.

Table 4

Comparison of dizziness handicap inventory (DHI) in the study groups at different duration from the start of the study till 12 months follow up

\begin{tabular}{lccccc}
\hline \multicolumn{2}{c}{$\begin{array}{c}\text { Duration of } \\
\text { evaluation }\end{array}$} & $\begin{array}{c}\text { VRT group } \\
(n=20)\end{array}$ & $\begin{array}{c}\text { Steroid group } \\
(n=20)\end{array}$ & $\begin{array}{c}\text { Combination group } \\
(n=20)\end{array}$ & P1 \\
\hline Baseline & Mean & 97.00 & 95.90 & 96.30 & 0.8 \\
\pm SD & 2.700 & 3.100 & 2.500 & & 0.054 \\
1 month & Mean & $22.10^{\mathrm{a}}$ & $25.90^{\mathrm{a}}$ & $20.80^{\mathrm{a}}$ & \\
\pm SD & 5.300 & 7.200 & 7.600 & & 0.09 \\
3 months & Mean & $15.20^{\mathrm{ab}}$ & $17.50^{\mathrm{ab}}$ & $14.80^{\mathrm{ab}}$ & \\
\pm SD & 3.400 & 4.600 & 4.30 & & \\
6 months & Mean & $10.80^{\mathrm{abc}}$ & $11.00^{\mathrm{abc}}$ & $10.10^{\mathrm{abc}}$ & 0.45 \\
\pm SD & 3.100 & 3.300 & 3.000 & & \\
12 months & Mean & $2.800^{\mathrm{abcd}}$ & $3.100^{\mathrm{abcd}}$ & $2.56^{\mathrm{abcd}}$ & 0.1 \\
\pm SD & 0.7000 & 0.8700 & 0.8300 & & \\
& $\mathrm{P} 2$ & $<0.001^{*}$ & $<0.001^{*}$ & $<0.001^{*}$ & \\
\hline
\end{tabular}

SD: standard deviation P: Probability *: significance $<0.05$. Test used: for comparison between groups one way ANOVA -test (P1) \& for comparison between time intervals repeated measures ANOVA followed by post-hoc Bonferroni (P2). ${ }^{a}$ : significance relative to baseline either in VRT group, steroid group or combination group. ${ }^{\mathrm{b}}$ : significance relative to 1 month either in VRT group, steroid group or combination group. ${ }^{c}$ : significance relative to 3 months either in VRT group, steroid group or combination group. ${ }^{\mathrm{d}}$ : significance relative to 6 months either in VRT group, steroid group or combination group. 


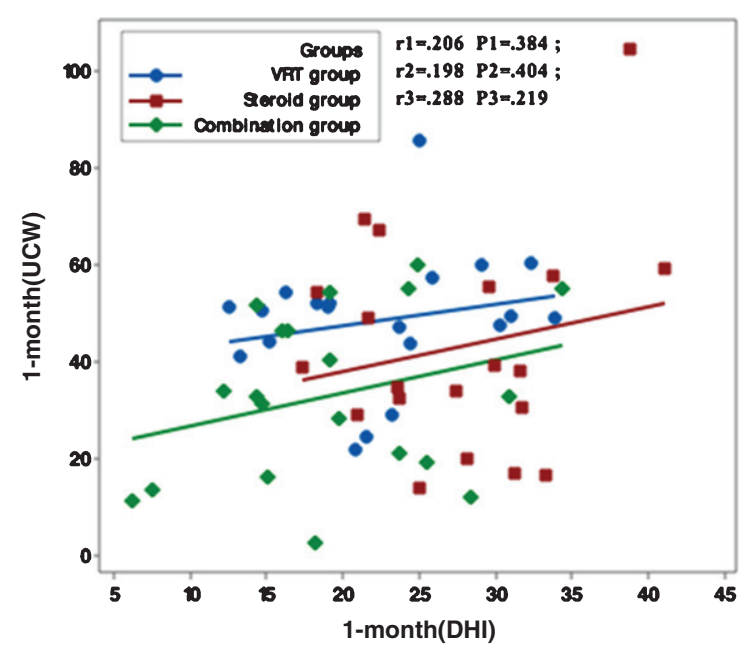

Fig. 2. Correlation between UCW and DHI in the study groups at one month from the start of the study. r: Pearson's correlation coefficient P: Probability *: significance $<0.05$.

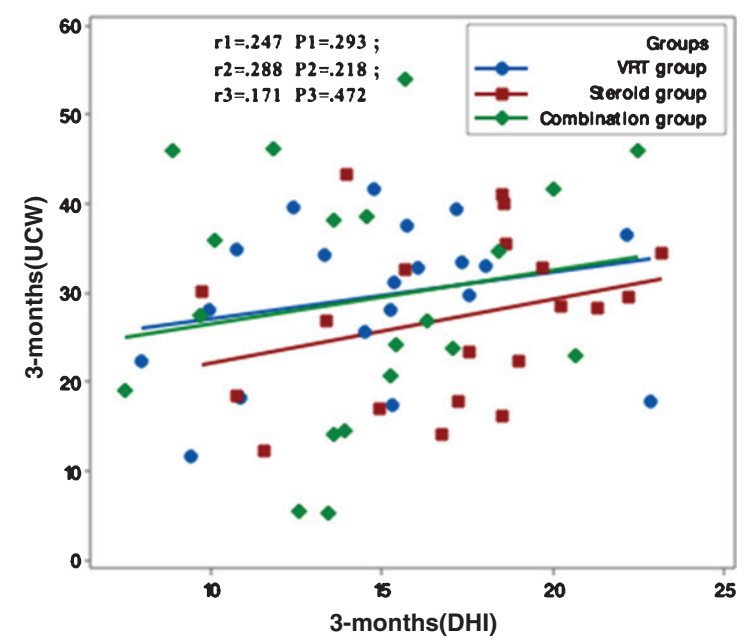

Fig. 3. Correlation between UCW and DHI in the study groups at 3 months from the start of the study. r: Pearson's correlation coefficient P: Probability *: significance $<0.05$.

\section{Discussion}

$\mathrm{VN}$ is a common disabling disease of the peripheral vestibular system. Till now, there is uncertainty on the best treatment, although several trials were carried out to compare different therapeutic strategies whether medical or physical. Different medications have been previously investigated to show their role in $\mathrm{VN}$ treatment. The role of corticosteroids in the treatment of $\mathrm{VN}$, on the basis of their role in the treatment of Bell's palsy, optic neuritis and sudden SNHL has been investigated. Strupp et al. [23] in

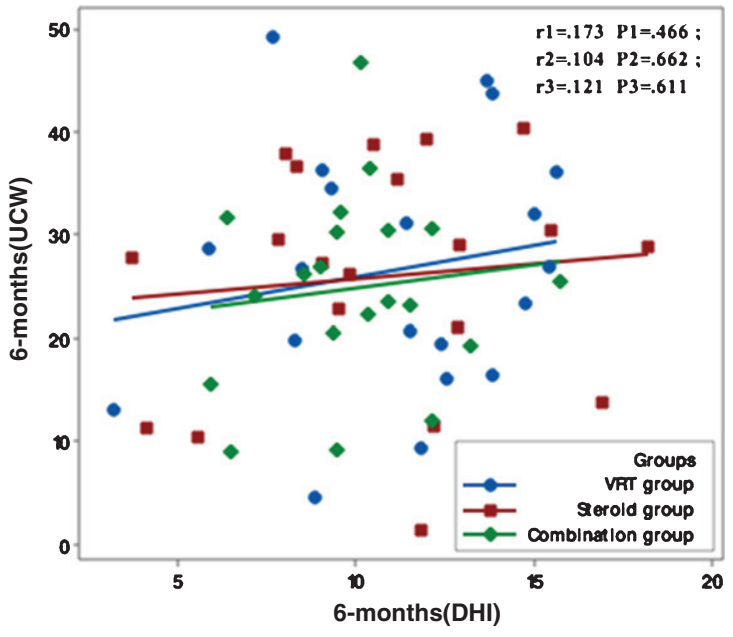

Fig. 4. Correlation between UCW and DHI in the study groups at 6 months from the start of the study. r: Pearson's correlation coefficient P: Probability *: significance $<0.05$.

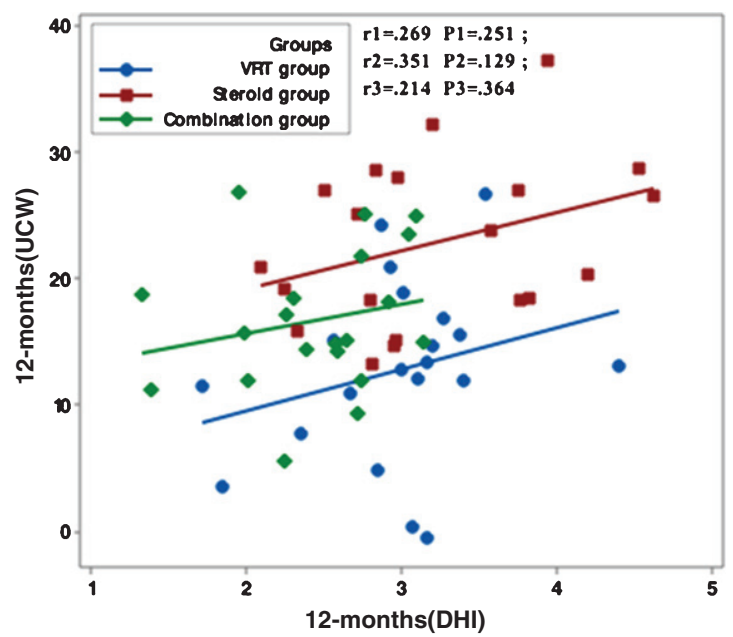

Fig. 5. Correlation between UCW and DHI in the study groups at 12 months from the start of the study. r: Pearson's correlation coefficient P: Probability *: significance $<0.05$.

a prospective randomized clinical trial used unilateral caloric weakness as a measure for the effect of steroids and antiviral drugs in $\mathrm{VN}$ treatment. The authors reported that steroids (methylprednisolone) have beneficial effects and significantly improved peripheral vestibular function although the authors depended on caloric test only as a measure of patient's improvement. In 2008, Shupak et al. [19], using both clinical and laboratory measure (ENG) for measuring patient's outcome, stated that steroid may be beneficial for patient with $\mathrm{VN}$ and enhance earlier recovery with no benefit for a long term outcome. 
A meta-analysis of all studies reviewing the value of steroid in VN patients reported beneficial effect with reference to caloric test without significant clinical improvement as regards patient symptoms [7]. Fishman et al. [5] reported insufficient evidence to use steroids in patients with VN. In more recent work [5], both vestibular exercises and steroids showed equal effects in long term prognosis of patients with $\mathrm{VN}$; however, steroids seem to have more benefits in early disease and enhance earlier recovery.

Yoo et al. [25] conducted a prospective randomized control study on vestibular neuritis patients in which the study group received steroids while the control group did not; vestibular exercises were performed in both groups. Extensive vestibular testing includes caloric irrigations, video Head Impulse Test (vHIT), and Sensory Organization Test (SOT) was carried out; DHI was reported at the start of the study; all tests were repeated at one and six months from the study. The study suggested that there were no differences in the improvement rates of objective as well as selfperceived handicap measures in both groups. This research recommended that steroid therapy might not provide additional benefit to $\mathrm{VN}$ patients at one and six months follow up.

In the present study, assessed patient's outcome both clinically (DHI) and at laboratory levels (VNG and (VEMP) and we compared steroids, VRT and the combination of both treatments. The results of the present study reveal that the use of steroids has no advantage over the use of VRT on long term follow up; however, it may have benefits in early stages and enhance early recovery both clinically and at laboratory levels as witnessed in follow up interval at one and three months mainly. These results are in accordance with the work performed by Goudakos et al. [8].

Compiling the results of both treatments in the combination group showed no better results than single treatment whether early or late in the disease follow up. The use of VRT in patient with VN has been previously reported and now, there is no doubt about its efficacy in VN patient as it reduces symptoms and limits the use of medications in these patients $[8,11]$.

Although the present study used caloric test and cVEMP response as a laboratory measure of labyrinthine recovery, the caloric test only assesses the low frequency response of the vestibular system $(0.002$ to $0.004 \mathrm{~Hz})$ and the function of the horizontal SCC. Much previous research reported that the recovery of vestibular system may be improved in the low frequency response but persistent asymmetry in the high frequency response may occur in patient with chronic VN. This conclusion is in line with the results proposed in the present study. Caloric response improved over the follow up period while the greatest improvements occurred early in the disease course.

One limitation of the present study is that the most recently available methods of assessing SCC and otolith function, such as video head impulse test that evaluates high frequency response of SCC and subjective visual vertical that expands evaluations of otolith function, were not employed; this may be recommended for further comparison between both low and high frequency response course of the vestibular system in patients with VN and expands the other aspects of complete disease resolution. However, Patel et al. [17] concluded that chronic symptoms of dizziness following $\mathrm{VN}$ were not correlating with the high velocity VOR of the single or combined semicircular canals affection. Similarly, Cousins et al. [3] reported that $\mathrm{VN}$ recovery is not predicted by visual vertical findings, however, increased visual dependence (assessed by the influence of a rotating visual background upon the visual vertical), as well as questionnaire measures of autonomic arousal, anxiety/depression and fear of bodily sensations, are effective predictors of clinical outcome in vestibular neuritis.

The present study emphasized the previously reported lack of correlation between clinical recovery and laboratory measures of the vestibular system namely caloric test; this may support the limited usefulness of caloric test only as a follow up measure in patient with $\mathrm{VN}$. The present study assumes that improvement of vestibular functions in VN depends on multiple factors such as the central compensation and the neural plasticity which differs from person to person regardless of the management protocol.

\section{Conclusion}

VRT and corticosteroids are similarly effective in patients with VN. Corticosteroids may accelerate recovery of $\mathrm{VN}$, with no more beneficial role in the long-term prognosis of the disease. Combined use of VRT and corticosteroids seems to have no other benefit in patients with $\mathrm{VN}$ than the use of single line of therapy. Laboratory measures of vestibular system function should be interpreted with caution as indicators of disease resolution. 


\section{Compliance with ethical standards}

Authors declare that they have no conflict of interest. For ethical approval, all procedures performed were in accordance with the ethical standards of Mansoura University and the National Research Committee and with the 1964 Helsinki Declaration and its later amendments. Informed consent was obtained from all individual participants included in the study.

\section{References}

[1] J. Bergenius and O. Perols, Vestibular neuritis: A follow-up study, Acta otolaryngologica 119 (1999), 895-899.

[2] F. Brusaferri and L. Candelise, Steroids for multiple sclerosis and optic neuritis: a meta-analysis of randomized controlled clinical trials, J Neurol 247 (2000), 435-442.

[3] S. Cousins, D. Kaski, N. Cutfield, Q. Arshad, H. Ahmad, M.A. Gresty, B.M. Seemungal, J. Golding and A.M. Bronstein, Predictors of clinical recovery from vestibular neuritis: A prospective study, Ann ClinTransl Neurol 22 4(5) (2017), 340-346.

[4] M. El-Gohary, N. Kamal and A. Abdel-Maksoud, Vestibular testing at high frequency range. Unpublished M.Sc. thesis 2000, Ain Shams Fac of med, Cairo, Egypt.

[5] J.M. Fishman, C. Burgess and A. Waddell, Corticosteroids for the treatment of idiopathic acute vestibular dysfunction (vestibular neuritis), Cochrane Database Syst Rev (5) 2011. CD008607. DOI: 10.1002/14651858.CD008607.pub2.

[6] J.K. Goudakos and K.D. Markou, Corticosteroids vs Corticosteroids Plus Antiviral Agents in the Treatment of Bell Palsy: A Systematic Review and Meta-analysis, Arch Otolaryngol Head Neck Surg 135 (2009), 558-564.

[7] J.K. Goudakos, K.D. Markou, V. Franco-Vidal, V. Vital, M. Tsaligopoulos and V. Darrouzet, Corticosteroids in the treatment of vestibular neuritis: A systematic review and meta-analysis, OtolNeurotol 31 (2010), 183-189.

[8] J.K. Goudakos, K.D. Markou, G. Psillas, V. Vital and M. Tsaligopoulos, Corticosteroids and Vestibular Exercises in Vestibular Neuritis Single-blind Randomized Clinical Trial, JAMA Otolaryngol Head Neck Surg 140(5) (2014), 434-440.

[9] A. Greco, G. Macri, A. Gallo, M. Fusconi, A. De Virgilio, G. Pagliuca, C. Marinelli and M. de Vincentiis, Is Vestibular Neuritis an Immune Related Vestibular Neuropathy Inducing Vertigo? J of Immunol Res 2014 (2014), 459048.
[10] G.M. Halmagyi and I.S. Curthoys, A clinical sign of canal paresis, Arch Neurol 45 (1988), 737-739.

[11] S.L. Hillier and M. McDonnell, Vestibular rehabilitation for unilateral peripheral vestibular dysfunction, Cochrane Database Syst Rev (2) 2011.

[12] Y. Imate and T. Sekitani, Vestibular compensation in vestibular neuronitis. Long-term follow-up evaluation, ActaOtolaryngol 113 (1993), 463-465.

[13] G.P. Jacobson and C.W. Newman, The development of the Dizziness Handicap Inventory, Arch Otolaryngol Head Neck Surg 116 (1990), 424-427.

[14] L.B.W. Jongkees, Value of the caloric test of the labyrinth, Arch Oto- laryngol 48 (1948), 402-417.

[15] M. McDonnell and L.M. Hillier, Vestibular Rehabilitation for Unilateral Peripheral Vestibular Dysfunction, Cochrane Database Syst Rev (1) 2015.

[16] Y. Okinaka, T. Sekitani, H. Okazaki, M. Miura and T. Tahara, Progress of caloric response of vestibular neuronitis, ActaOtolaryngol Suppl 503 (1993), 18-22.

[17] M. Patel, Q. Arshad, R.E. Roberts, H. Ahmad and A.M. Bronstein, Chronic symptoms after vestibular neuritis and the high velocity vestibulo-ocular reflex, OtolNeurotol 37(2) (2016), 179-184.

[18] J.M. Schweinfurth, S.M. Parnes and M. Very, Current concepts in the diagnosis and treatment of sudden sensorineural hearing loss, Eur Arch Otorhinolaryngol 253 (1996), 117-121.

[19] A. Shupak, A. Issa, A. Golz, Margalit Kaminer and I. Braverman, Prednisone treatment for vestibular neuritis, OtolNeurotol 29 (2008), 368-374.

[20] A. Shupak, Z. Nachum, Y. Stern, D. Tal, A. Gil and C.R. Gordon, Vestibular neuronitis in pilots: Follow-up results and implications for flight safety, Laryngoscope 113 (2003), 316-321.

[21] M. Strupp and T. Brandt, Vestibular neuritis, Semin Neurol 29 (2009), 509-519.

[22] M. Strupp, K. Hüfner, R. Sandmann, A. Zwergal, M. Dieterich, K. Jahn and T. Brandt, Central oculomotor disturbances and nystagmus: A window into the brainstem and cerebellum, DtschArzteblInt 108 (2011), 197-204.

[23] M. Strupp, V.C. Zingler, V. Arbusow, D. Niklas, K.P. Maag, M. Dieterich, S. Bense, D. Theil, K. Jahn and T. Brandt, Methylprednisolone, valacyclovir, or the combination for vestibular neuritis, N Engl J Med 351 (2004), 354-361.

[24] P.P. Vidal, C. De Waele, N. Vibert and M. Mühlethaler, Vestibular compensation revisited, Otolaryngol Head Neck Surg 119 (1998), 34-42.

[25] M.H. Yoo, C.J. Yang, S.A. Kim, M.J. Park, J.H. Ahn, J.W. Chung and H.J. Park, Efficacy of steroid therapy based on symptomatic and functional improvement in patients with vestibular neuritis: A prospective randomized controlled trial, Eur Arch Otorhinolaryngol 274 (2017), 2443-2451. 